\title{
Interrogating mechanisms of ssDNA binding to a viral HUH-endonuclease by alanine scanning of an electrostatic patch
}

Andrew Nelson, Kassidy Tompkins, Maria Paz Ramirez, Wendy R. Gordon

University of Minnesota, Department of Biochemistry, Molecular Biology, and Biophysics, Minneapolis, MN 55409

Address correspondence to: wrgordon@umn.edu

\begin{abstract}
:
$\mathrm{HUH}$ endonucleases (dubbed "HUH-tags") are small protein domains capable of forming covalent adducts with ssDNA in a sequence-specific manner. Because viral HUH-tags are relatively small, react quickly, and require no chemical modifications to their ssDNA substrate, they have great value as protein fusion tags in biotechnologies ranging from genetic engineering to single-molecule studies. One of the greatest assets of these tags is sequence-specificity to their unique, native Ori sequence in vivo, introducing the possibility of using multiple HUH-tags in multiplexed "one-pot" reactions. However, their mechanism of ssDNA sequence binding and specificity is poorly understood, and there is noted cross-reactivity between tags of closely related species. In order to understand the mechanism of ssDNA binding, we performed an alanine scan along a positively-charged patch of one such $\mathrm{HUH}$-tag, replication-associated protein from Wheat Dwarf Virus (WDV Rep), and characterized the enzymatic activity in both the rate and extent of the reaction. In molecular beacon Stopped-Flow experiments, single point
\end{abstract}


mutants of WDV showed a more than $60 \%$ decrease in reaction rate constant, and gel shift assays showed an almost complete lack of activity for some variants for single nucleotide ssDNA substitutions. In all, these findings help allow us to highlight key interactions in WDV-ssDNA binding, and we gain further insight into potential rational engineering of $\mathrm{HUH}-e n d o n u c l e a s e s$ to bind desired sequences of DNA.

\section{Introduction:}

The replication-associated domain of Wheat Dwarf Virus (WDV Rep) falls within a family of viral and bacterial phosphodiesterases known as HUH endonucleases (Yan and Fei 2007). In viruses, HUH endonucleases initiate rolling circle replication of the viral genome by recognizing the conserved nucleotide sequence of a ssDNA hairpin structure at its origin of replication and introducing a 5'-nick to the phosphate backbone. This nick is facilitated by the histidine-hydrophobic-histidine $(\mathrm{HUH})$ motif of the protein, which coordinates a metal ion to stabilize a nucleophilic tyrosine. In vivo, this is a transient reaction, and the free 3 '-end of the template strand re-ligates with the 5'-end at the end of genomic replication, releasing the Rep protein. In contrast, this reaction forms a robust phosphotyrosine adduct in vitro, and this protein-DNA conjugate is stable even in denaturing conditions (Chandler et al. 2013; Lovendahl, Hayward, and Gordon 2017; Heyraud-Nitschke et al. 1995). Thus, HUH endonucleases pose an attractive option for creating stable protein-DNA linkages in a variety of biotechnology applications, for example improving CRISPR-Cas9 precise gene editing (Aird et al. 2018) and tethering proteins of interest in DNA origami applications (Sagredo et al. 2016). Moreover, each $\mathrm{HUH}$ endonuclease recognizes a distinct sequence at its native origin of replication, resulting in a sequence-specific covalent adduct. Interestingly, HUH endonucleases derived from bacteria tend to be larger and less efficient at forming covalent adducts, but with more sequence 
specificity than their viral counterparts. Viral HUH endonucleases are small and form efficient covalent adducts, but their origin of replication sequences display more similarity, introducing the possibility of cross-reactivity. Understanding the molecular basis for single-stranded DNA recognition by viral $\mathrm{HUH}$ endonucleases offers the potential to engineer the viral HUH endonuclease to recognize desired DNA sequences, enhancing their multiplexing capacity. However, there are no crystal structures of $\mathrm{HUH}$-endonucleases derived from viruses in complex with DNA. Thus we aimed to probe the requirements for ssDNA binding to the $\mathrm{HUH}$-endonuclease domain from WDV by systematically mutating amino acids in a large, positively charged patch to alanine. The resulting variants were measured for effects on the kinetics of endonuclease activity, protein stability, and tolerance of nucleotide changes in the ssDNA substrate.

\section{Results and Discussion:}

We first identified electrostatic patches on WDV Rep using APBS electrostatics generation in Pymol and mapped them onto the recent crystal structure- pdb id: 6Q1M (Fig 1 A). We observed a large, positively charged patch in the vicinity of the catalytic residues (blue residues in Fig 1B comprising $\mathrm{H} 59$ and $\mathrm{H} 61$ involved in putative metal coordination and Y106, the site of phosphotyrosine bond formation) and reasoned that it might play some role in the binding and recognition of ssDNA. We mutated 11 amino acids (purple residues in Fig 1B) in this patch to alanine using site-directed mutagenesis, and these residues are colored yellow in the multiple sequence alignment of several viral HUH endonucleases shown in Fig $1 \mathrm{C}$. It should be noted that several of the amino acids mutated are highly conserved while some are not. It is possible that these residues which are not conserved convey sequence specificity for WDV 
Rep which is exclusive from other geminiviral HUH endonucleases. Moreover, though the residues are clustered in a patch on the surface of the protein, they are spread across the protein in sequence space. Previous research has suggested that this patch is necessary for nuclear localization of other geminiviral Rep proteins, and it may serve bifunctionally to bind the negative charge on ssDNA as well (Maio et al. 2019).

After expression and purification of these proteins using standard protocols, we used several assays to measure how the mutations affected aspects of HUH endonuclease activity. HUH endonuclease activity involves at least three steps: DNA binding, DNA cleavage, and DNA-adduct formation with the 5' end of the cleaved DNA (Fig 2A). We first used a 26-nt DNA oligo containing the canonical origin of replication sequence in an SDS-PAGE gel shift assay to measure the extent of covalent adduct formed by the wildtype protein compared to the alanine mutants. The reactions were performed under "standard" HUH-tag reaction conditions (10x DNA, 50mM Hepes 8, 50mM NaCl, $1 \mathrm{mM} \mathrm{MnCl2;} \mathrm{Lovendahl} \mathrm{et} \mathrm{al.} \mathrm{2017)} \mathrm{at} 37^{\circ} \mathrm{C}$ for 30 minutes, and band volumes were quantified to determine covalent adduct formation. The data displayed in Figure 2B shows the efficiency of covalent adduct formation of all of the mutants with a 26-nt oligo harboring the "canonical" origin of replication sequence of geminiviruses. Surprisingly, the percent covalent adduct formation was not affected by mutation of the electrostatic patch. In order to assess if the kinetics of the WDV alanine mutant cleavage activity is altered as compared to the WT WDV, we used a molecular beacon, stopped-flow assay (Fig 2C). We devised a pseudo-first order scheme mixing 10x excess oligo substrate harboring the geminivirus canonical target sequence substrate flanked by a quencher and fluorescein mixed with WDV recombinant protein (standard buffer conditions). Single-turnover cleavage of the 
labeled oligo results in a positive readout of increased fluorescence monitored over the course of the reaction. We fit the kinetic curves using DataPro Viewer to a triple exponential (SI) (insert eq.) and displayed the fastest rate constant, k2, associated with cleavage. The other two rate constants may be partially describing binding and release of the substrate. All WDV alanine mutants showed decreased cleavage rates as compared to the WT WDV, which had a rate constant $(\mathrm{k})$ of $0.28 \mathrm{~s}^{-1}$. Notably, the two most $\mathrm{N}$-terminally positioned arginine mutants, R8A and R10A, had over a $50 \%$ decrease in cleavage. The three most C-terminally positioned mutants, N74, N76A, and K98A, along with the double mutant K67/98A, had very significant decreases in cleavage rates as well.

We next used Differential Scanning Fluorimetry to measure how the mutations affected the stability of the $\mathrm{HUH}$-endonuclease to aid in deconvoluting the effects on DNA binding activity due to lost interactions versus destabilization of the protein. Shown is the average melting temperature of each mutant, computed from the second derivative curve of 4-8 replicate experiments (Fig 3). Several mutations showed up to 3 degrees decrease in melting temperature, and two mutants even showed enhanced stability. This suggests that the slower rates of cleavage of some of the mutants could, in part, be due to decreased protein stability.

Finally, we measured the effect of mutations on binding non-canonical DNA oligo sequences to tease apart the potential roles of residues on DNA binding versus DNA specificity. Gel shift assays show the covalent adduct formation of each WDV variant for single nucleotide substitutions of the canonical 26-nt target sequence starting from the cleavage site and moving towards the 5' (positions -1 through -6). Generally, most mutants had little to no change in covalent adduct formed in a five-minute reaction. However, the variants, R8A, R10A, N39A, and 
K98A showed noticeably decreased reactivity to nucleotide substitutions in positions $-1,-4,-5$, and -6 .

Overall, the R8A and K98A mutants showed the most significant effects on activity with ssDNA with little to no changes to protein stability. Given that these are also highly conserved amino acids, it is likely that these residues are critical for binding DNA substrates, perhaps correctly positioning ssDNA for cleavage. Other, less conserved mutants showed differential effects on ssDNA binding activity, perhaps suggesting more subtle roles in dictating sequence specificity.

It should be noted that the substitutions which were chosen for the gel shift assay are not exhaustive. Future studies would benefit from current, next-generation sequencing methods to observe the full profile of sequence specificity for each variant. Furthermore, NMR studies may highlight WDV's base interactions with DNA in a more sensitive, precise way than targeted mutagenesis. Inevitably, a crystal structure of WDV complexed to its ssDNA target would be most enlightening, and it would introduce the opportunity to rationally design the sequence-specificity of WDV. Our results provide a first step towards understanding the recognition of SSDNA by viral HUH endonucleases.

\section{Materials and Methods:}

\section{Homology Modeling and Mutant Design}

We initially designed our mutations based on a homology model of WDV. WDV homology model was generated using SWISS-MODEL(Waterhouse et al. 2018) with the NMR structure of Rep of tomato yellow leaf curl virus (PDB: 115i(Campos-Olivas et al. 2002)) as a template. The generated structure with the lowest QMEAN score was downloaded and opened in PyMol (Benkert, Biasini, and Schwede 2011; DeLano 2002). During these studies, we 
obtained the crystal structure of apo WDV and used the structure in Figure 1. The surface electrostatics of the model was generated using the APBS electrostatics plugin in PyMol, and positively charged residues within the apparent electropositive patch were selected for mutagenesis.

\section{Primers}

All mutagenizing primers were purchased from IDT. Sequences are as follows:

\begin{tabular}{|c|c|}
\hline Primer & Sequence \\
\hline K14A-->F & TATAGCGCGTACCTGTTTCTGACCTATCCGCAGTGTAC \\
\hline K14A-->R & CAGGTACGCGCTATAAACACGAAAACGCGGTGTGC \\
\hline K40A-->F & CTGAACGCGTATGAACCGCTGTATATTGCAGCAGTTCG \\
\hline K40A-->R & TTCATACGCGTTCAGCAGGGTACGCAGGCTATC \\
\hline K67A-->F & CAGAATGCGCTGCGTGCAAGCATTACCAATCCG \\
\hline K67A-->R & ACGCAGCGCATTCTGAACCAGAACATGCAGATGCGG \\
\hline K98A-->F & GCAGCCGCGGATTGTAATCAGGTGCGTGATTATATCACCAAAGAAG \\
\hline K98A-->R & ACAATCCGCGGCTGCCTGAATATTCGGATGAAAAATGC \\
\hline N39A-->F & CTGCTGGCGAAATATGAACCGCTGTATATTGCAGCAGTTCG \\
\hline N39A-->R & ATATTTCGCCAGCAGGGTACGCAGGCTATCC \\
\hline N66A-->F & GTTCAGGCGAAACTGCGTGCAAGCATTACCAATCCG \\
\hline N66A-->R & CAGTTTCGCCTGAACCAGAACATGCAGATGCGGAC \\
\hline N74A-->F & ATTACCGCGCCGAATGCACTGAATCTGCGTATGG \\
\hline N74A-->R & ATTCGGCGCGGTAATGCTTGCACGCAGTTTATTCTGAAC \\
\hline N76A-->F & AATCCGGCGGCACTGAATCTGCGTATGGATACCAGTC \\
\hline N76A-->R & CAGTGCCGCCGGATTGGTAATGCTTGCACGCAG \\
\hline R10A-->F & CGTTTTGCGGTTTATAGCAAATACCTGTTTCTGACCTATCCGC \\
\hline R10A-->R & ATAAACCGCAAAACGCGGTGTGCTGCTGC \\
\hline
\end{tabular}




\begin{tabular}{|l|l|}
\hline R69A-->F & AAACTGGCGGCAAGCATTACCAATCCGAATGCACTG \\
\hline R69A-->R & GCTTGCCGCCAGTTTATTCTGAACCAGAACATGCAGATGCG \\
\hline R8A-->F & ACACCGGCGTTTCGTGTTTATAGCAAATACCTGTTTCTGACCTATCC \\
\hline R8A-->R & ACGAAACGCCGGTGTGCTGCTGCTTGCC \\
\hline
\end{tabular}

\section{Protein Expression}

A pTD68 expression vector containing the coding sequence for residues 1-137 of WDV RepA (UniProt ID: P06847) attached to an N-terminal 6xHIS-SUMO tag was used for this study. Mutations to the RepA gene were introduced using In-Fusion primers (Takara) designed to contain the mutant Ala codon within the 15-bp homology arm (Supplemental For Primers). PCR amplification on these primers was done using CloneAmp HiFi PCR Premix (Clontech) and original template DNA was digested by Dpnl. Sequence-confirmed, mutant plasmids were transformed into competent Escherichia coli (E. coli) BL21-DE3 cells and grown in 1L cultures of LB broth supplemented with $100 \mathrm{ng} / \mathrm{mL}$ ampicillin. Cultures were grown at $37^{\circ} \mathrm{C}$ to an $\mathrm{OD}_{600}$ of 0.8 before induction by $500 \mu \mathrm{M}$ isopropyl $\beta$-D-1-thiogalactopyranoside (IPTG). Induced cultures were grown overnight at $18^{\circ} \mathrm{C}$ then pelleted by centrifugation at $4000 \times \mathrm{G}$ for $30 \mathrm{~min}$. After resuspending in $6 \mathrm{xHIS}$ lysis buffer (50mM tris, 250mM NaCl, $1 \mathrm{mM}$ EDTA, pH 7.5), cells were lysed by sonication, and cellular debris was pelleted by centrifugation at $25000 \times \mathrm{G}$ for $30 \mathrm{~min}$. Soluble protein was batch-bound to $\mathrm{Ni}^{2+}-\mathrm{NTA}$ resin and washed by 10 column-volumes of $6 \mathrm{xHIS}$ wash buffer (50mM tris, $250 \mathrm{mM} \mathrm{NaCl}, 1 \mathrm{mM}$ EDTA, $30 \mathrm{mM}$ imidazole, pH 7.5). Protein was eluted in $6 \mathrm{xHIS}$ elution buffer (50mM tris, $150 \mathrm{mM} \mathrm{NaCl}, 1 \mathrm{mM}$ EDTA, 300mM imidazole, $\mathrm{pH} 7.5)$, and imidazole was removed by size-exclusion chromatography through a Bio-Rad SEC70 column (Buffer: 50mM tris, 150mM NaCl, 1mM EDTA, pH 7.5). 


\section{Differential Scanning Fluorimetry}

SYPRO Orange dye (Thermo Fisher Scientific; 5000x in DMSO) was diluted 1:400 in experimental buffer (20mM HEPES, $500 \mathrm{mM} \mathrm{NaCl}, \mathrm{pH} 7.4) .30 \mu \mathrm{L}$ of $2 \mu \mathrm{M}$ proteins in experimental buffer were combined with $5 \mu \mathrm{L}$ of the diluted dye in hard-shell, low-profile, 96-well, PCR plates (Bio-Rad). Plates were centrifuged at 4000 x G for 2 minutes before immediate analysis in a CFX96, real-time, qPCR machine (Bio-Rad). Proteins were held at 20 ${ }^{\circ} \mathrm{C}$ for $2 \mathrm{~min}$, before the temperature was ramped to $90{ }^{\circ} \mathrm{C}$ at a rate of $0.5^{\circ} \mathrm{C} / 30$ sec. Plate RFUs were scanned every $30 \mathrm{sec}$ using the FRET channel, and melting temperatures were determined from the second derivative of the RFU vs. Temperature curve.

\section{Circular Dichroism}

Confirmation of DSF results were performed using thermal denaturation in circular dichroism (CD). Each purified protein was centrifuged at $14,000 \times \mathrm{G}$ for 10 minutes at $4{ }^{\circ} \mathrm{C}$ and the supernatant diluted to $0.35 \mathrm{mg} / \mathrm{mL}$ using PBS. Absorption at $222 \mathrm{~nm}$ was acquired with a Jasco J-815 spectropolarimeter, temperature controlled by a Peltier device. Changes in the absorption at $222 \mathrm{~nm}$ were acquired at a temperature gradient of $1{ }^{\circ} \mathrm{C} / \mathrm{min}$ temperature intervals from $20-90{ }^{\circ} \mathrm{C}$, and the characteristic ellipticity at alpha-helical wavelength $(\theta 222)$ recorded. Molar ellipticity, $[\theta]$, was calculated using the following equation: $[\theta]=\theta /(10 c /)$ where $c$ is the molar concentration of the sample (mole/L) and $/$ is the path-length in $\mathrm{cm}$. Molar ellipticity (with units of degrees, cm squared per decimole) was plotted against wavelength for the circular dichroism (CD) spectra. Ellipticity at $222 \mathrm{~nm}(\theta 222)$ was normalized, plotted against temperature, and fit by regression analysis in Sigma Plot (Systat Software, Inc.) using equations for two-state unfolding.

\section{Gel Shift Assay}


In HUH reaction buffer (50 mM HEPES, $50 \mathrm{mM} \mathrm{NaCl}, 1 \mathrm{mM} \mathrm{MnCl}$, $\mathrm{pH}$ 8), $3 \mu \mathrm{M}$ proteins were combined with $6 \mu \mathrm{M}$ 26-nucleotide ssDNA sequences, and the reaction was allowed to proceed for $5 \mathrm{~min}$ at $37^{\circ} \mathrm{C}$. Reactions were stopped with $4 x$ SDS loading buffer $+2 \%(v / v)$ $\beta$-mercaptoethanol and heat denatured at $95^{\circ} \mathrm{C}$ for five minutes. Reactions were analyzed by gel electrophoresis on $4-20 \%$ polyacrylamide gels stained with coomassie blue for 30 min then de-stained in $\mathrm{ddH}_{2} \mathrm{O}$ overnight. Percent adduct was quantified using automatic band detection in Image Lab software (Bio-Rad).

\section{Stopped-Flow Kinetics}

Molecular Beacon Stopped-flow HUH-tag Kinetics

Description: HUH-tag single turnover cleavage kinetics were characterized using a ssDNA molecular beacon assay on a standard stopped-flow instrument equipped with a fluorescence detector. The stopped-flow instrument (Applied Photophysics SX20) monochromater was set to $490 \mathrm{~nm}$ and $1.5 \mathrm{~mm}$ slit widths. Drive syringes were loaded with HPLC purified oligo substrate containing the geminivirus ori sequence and a 5' iowa black-FQ quencher and a 3' 3-6 fluorescein ( 5'IABkFQ/CGTATAATATTACCGGATGGCCGCGC/36-FAM/, IDT) or $100 \mathrm{nM}$ HUH-tag each in reaction buffer, pH 8. A PMT detector (Hammamatsu) and $500 \mathrm{~nm}$ longpass filter (Edmund Optics) monitored cleavage of the substrate immediately following rapid mixing in the flow chamber for a duration of 2 minutes, and 1000 data points were collected in the logarithmic setting.

\section{Figures:}


C
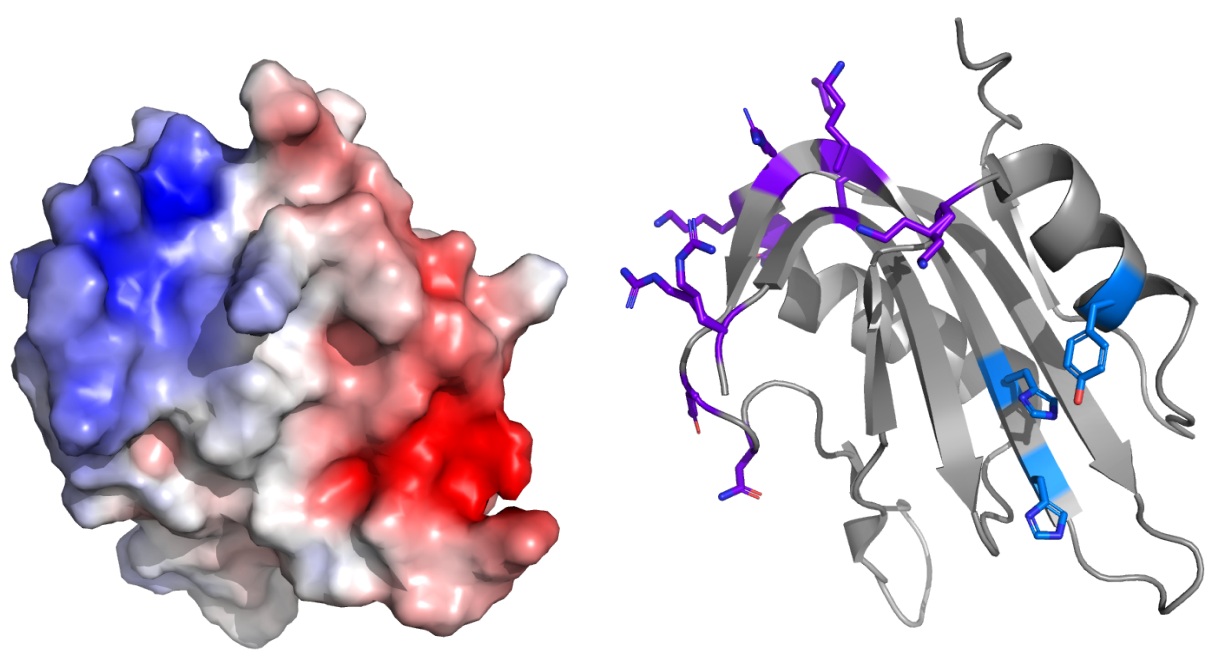

\begin{tabular}{l} 
Conservation: \\
WDV_Rep \\
CPV_Rep \\
TYLCV_Rep \\
PCV2_Rep \\
DCV_Rep \\
Consensus aa: \\
Consensus_ss: \\
\hline \\
Conservation: \\
WDV_Rep \\
CPV_Rep \\
TYLCV_Rep \\
PCV2_Rep \\
DCV_Rep \\
Consensus aa: \\
Consensus ss: \\
\hline \\
Conservation: \\
WDV_Rep \\
CPV_Rep \\
TYLCV_Rep \\
PCV2_Rep \\
DCV_Rep \\
Consensus aa: \\
Consensus ss:
\end{tabular}

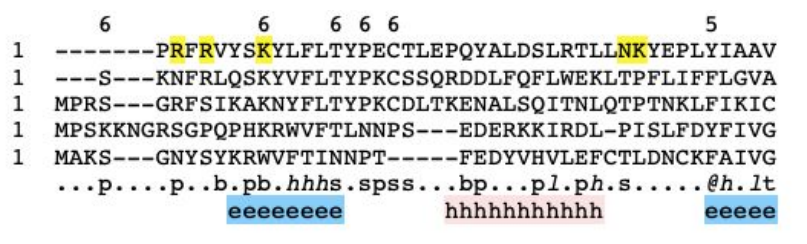

43
44
47
46
42

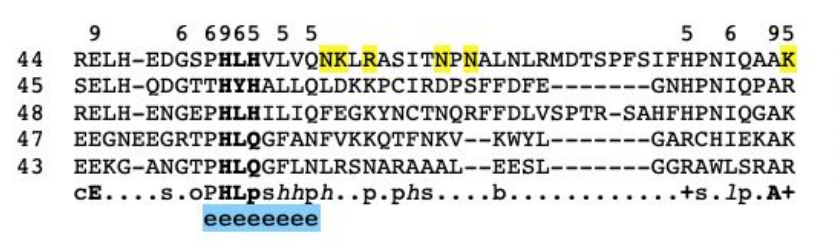

92
86
95
87
82

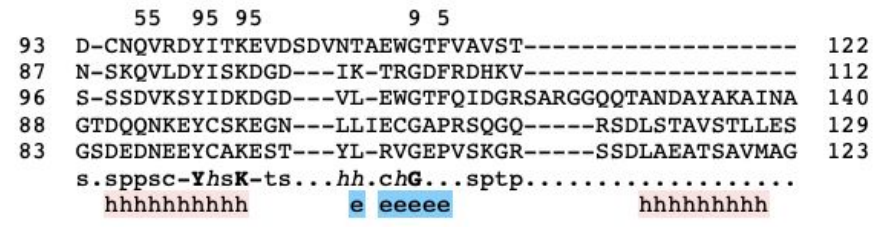

Figure 1. Alanine scanning of electrostatic patch. A. Surface representation of the WDV endonuclease with negatively charged regions colored red and positively charged regions colored blue. B. Side chains of amino acids of interest shown as ball and stick. Amino acids in the positively charged patch colored purple. Catalytic residues colored blue. C. Multiple sequence alignment of several viral HUH-endonucleases. Residues mutated in this study are highlighted yellow. Secondary structure depicted below alignment. Sequence conservation depicted by numbers above the sequence. 
A

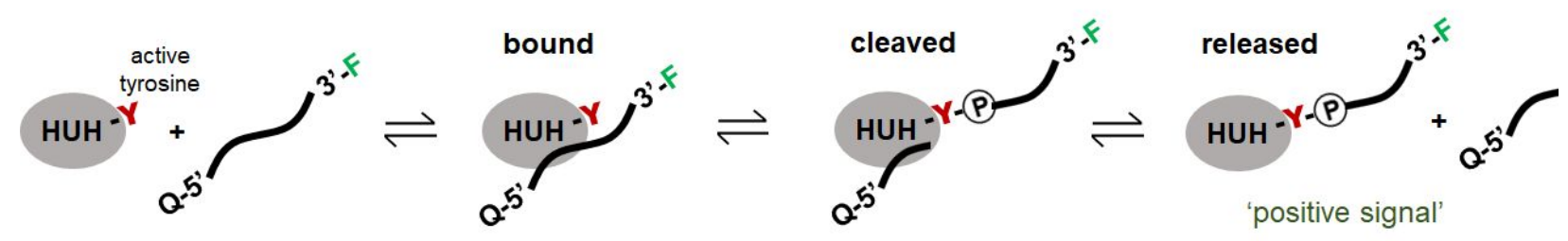

B

C

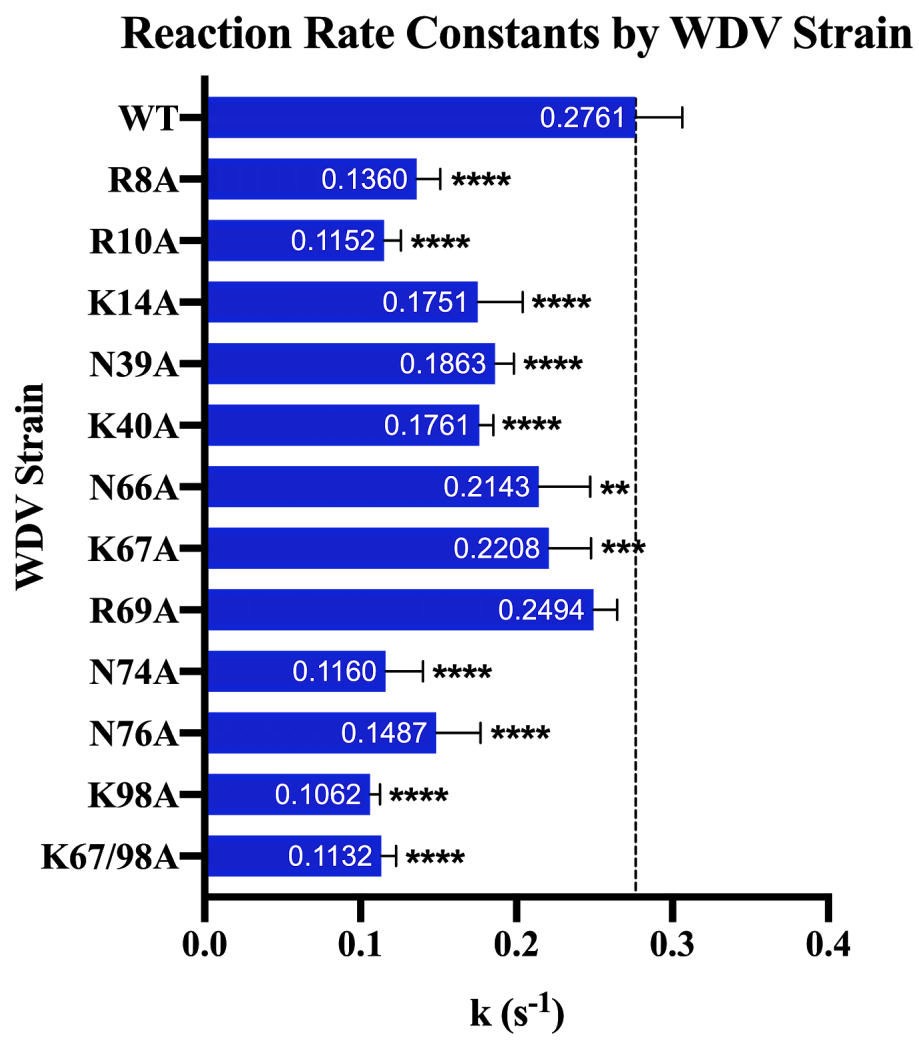

Figure 2. Mutation effect of WDV activity with canonical ssDNA sequence. A. Reaction scheme of HUH endonuclease formation of covalent adduct with ssDNA. B. Gel shift assay of WT and mutant WDV with ssDNA. C. Stopped-flow kinetics assay depicting DNA cleavage reaction of WDV and mutants with a molecular beacon ssDNA substrate. Statistical significance was calculated using ANOVA in Prism. 


\section{Melting Temperature by WDV Strain}

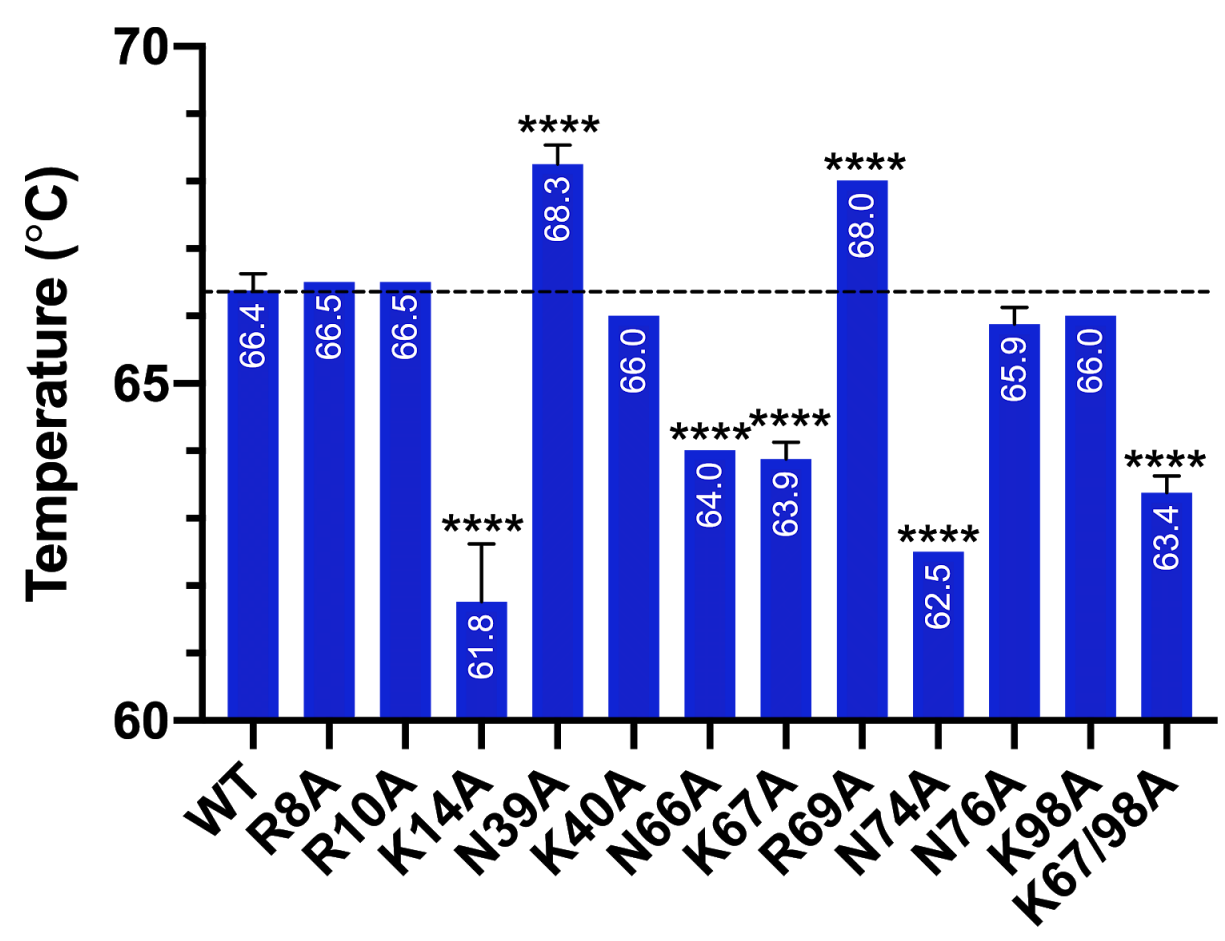

Figure 3. Stability of WDV and alanine mutants measured by Differential Scanning Fluorimetry. 


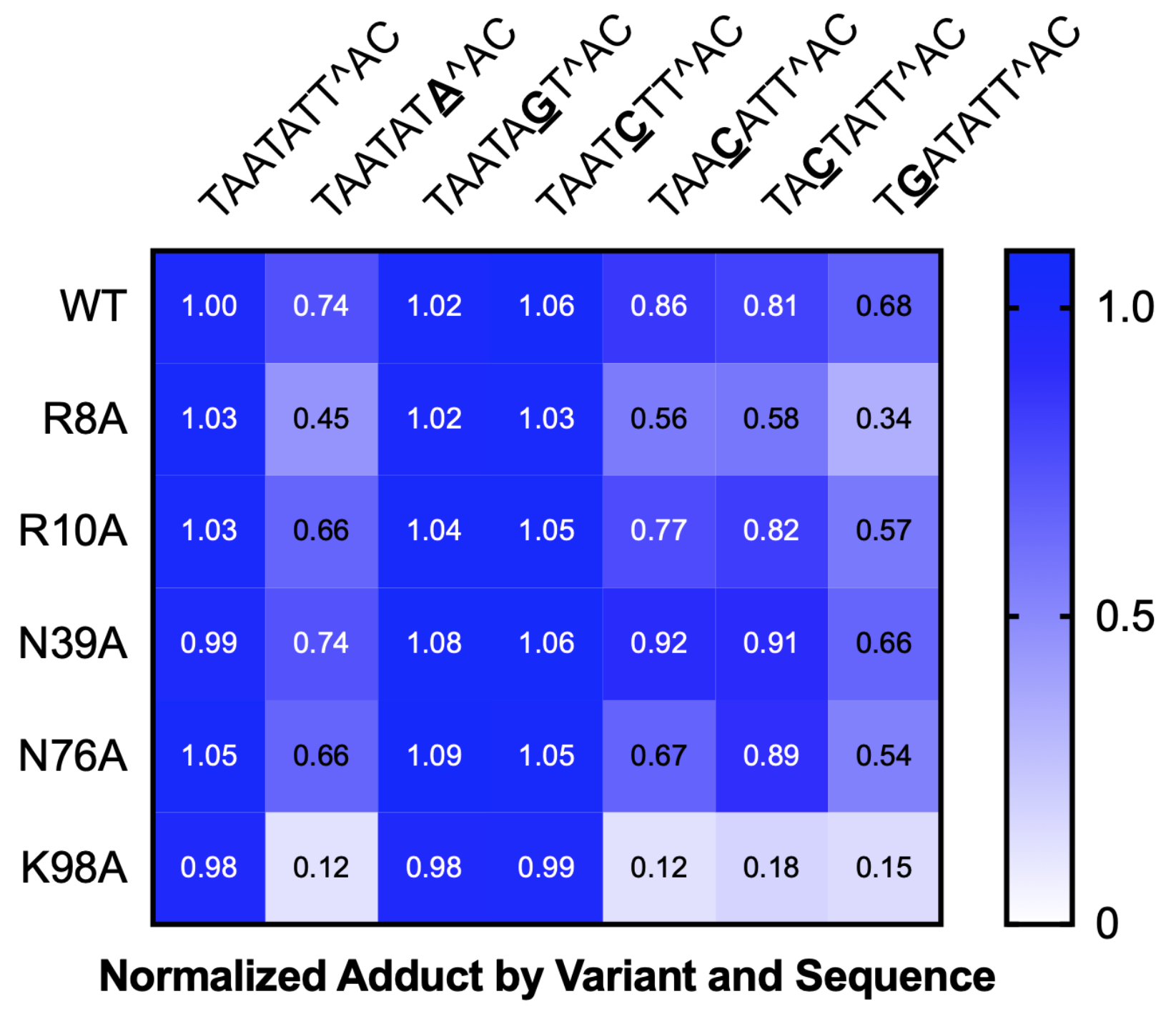

Figure 4. Covalent adduct formation by WDV and mutants upon reaction with altered ssDNA sequences. Gel shift assays were performed, and adduct formation was quantified using gel imaging software and normalized to the reaction of wildtype WDV with wtDNA sequence. 


\section{References cited:}

Aird, Eric J., Klaus N. Lovendahl, Amber St Martin, Reuben S. Harris, and Wendy R. Gordon. 2018. "Increasing Cas9-Mediated Homology-Directed Repair Efficiency through Covalent Tethering of DNA Repair Template." Communications Biology 1 (May): 54.

Benkert, Pascal, Marco Biasini, and Torsten Schwede. 2011. "Toward the Estimation of the Absolute Quality of Individual Protein Structure Models." Bioinformatics 27 (3): 343-50.

Campos-Olivas, Ramon, John M. Louis, Danielle Clerot, Bruno Gronenborn, and Angela M. Gronenborn. 2002. "The Structure of a Replication Initiator Unites Diverse Aspects of Nucleic Acid Metabolism." Proceedings of the National Academy of Sciences of the United States of America 99 (16): 10310-15.

Chandler, Michael, Fernando De La Cruz, Fred Dyda, Alison B. Hickman, Gabriel Moncalian, and Bao Ton-Hoang. 2013. "Breaking and Joining Single-Stranded DNA: The HUH Endonuclease Superfamily." Nature Reviews. Microbiology 11: 525-38.

DeLano, Warren Lyford. 2002. "PyMOL." virology.wisc.edu. http://virology.wisc.edu/acp/Classes/DropFolders/Drop660_lectures/2013_660/L01_PyMOL 2013r.pdf.

Heyraud-Nitschke, Françoise, Silke Schumacher, Jürgen Laufs, Sabine Schaefer, Jeff Schell, and Bruno Gronenborn. 1995. "Determination of the Origin Cleavage and Joining Domain of Geminivirus Rep Proteins." Nucleic Acids Research 23 (6): 910-16.

Lovendahl, Klaus N., Amanda N. Hayward, and Wendy R. Gordon. 2017. "Sequence-Directed Covalent Protein-DNA Linkages in a Single Step Using HUH-Tags." Journal of the American Chemical Society 139 (20): 7030-35.

Sagredo, Sandra, Tobias Pirzer, Ali Aghebat Rafat, Marisa A. Goetzfried, Gabriel Moncalian, Friedrich C. Simmel, and Fernando de la Cruz. 2016. "Orthogonal Protein Assembly on DNA Nanostructures Using Relaxases." Angewandte Chemie 55 (13): 4348-52.

Waterhouse, Andrew, Martino Bertoni, Stefan Bienert, Gabriel Studer, Gerardo Tauriello, Rafal Gumienny, Florian T. Heer, et al. 2018. "SWISS-MODEL: Homology Modelling of Protein Structures and Complexes." Nucleic Acids Research 46 (W1): W296-303.

Yan, Fei, and Y. A. N. Fei. 2007. "Transgenic Wheat Expressing Virus-Derived Hairpin RNA Is Resistant to Barley Yellow Dwarf Virus." HEREDITAS. https://doi.org/10.1360/yc-007-0097. 